\title{
A PROPENSÃO AO BIM NO CURSO DE ENG. CIVIL DURANTE ATIVIDADES REMOTAS NA PANDEMIA
}

DOI: 10.37702/2175-957X.COBENGE.2021.3741

José David Monte da Silva - davidmonte@alu.ufc.br

Universidade Federal do Ceará

Travessa Santo Antônio 86

63700-007 - Crateús - CE

Luis Felipe Cândido - luisfcandido2015@gmail.com

Universidade Federal do Ceará

BR $226 \mathrm{Km} 4$

63700-000 - Crateús - CE

Stelamaris Alves de Siqueira - asstelamaris@gmail.com

Universidade Federal do Ceará

Rua Firmino Rosa 1092

63700-028 - Crateús - CE

Resumo: A Modelagem da Informação na Construção, ou BIM (do Inglês Building Information Modelling), promove a digitalização das informações da construção para diversos usos em todo o ciclo de vida do empreendimento e sua aplicação requer novas competências dos profissionais da indústria de Arquitetura, Engenharia e Construção (AEC). Tal exigência provoca a necessidade de mudanças nos currículos dos cursos de formação do setor, que vêm ocorrendo de forma gradual e pouco eficiente. Especialmente no ano de 2020, marcado por uma pandemia global, a comunidade acadêmica precisou se adaptar ao ensino remoto, requerendo maior colaboração dos alunos em atividades avaliativas grupais. Nesse contexto, o BIM possui ferramentas de trabalho colaborativo que podem favorecer o trabalho remoto e colaborativo. Assim, este trabalho teve por objetivo analisar a propensão ao trabalho com BIM por alunos de graduação em engenharia civil. Tomou-se como objeto de estudos a elaboração de um projeto residencial desenvolvido remotamente no âmbito de uma disciplina em um curso de uma universidade federal no interior do estado do Ceará. Aplicou-se um formulário online após a conclusão do trabalho em causa tendo sido possível compreender as dificuldades dos alunos no processo, especialmente no tocante ao BIM e a dinâmica de colaboração das equipes. Verificou-se que a comunicação foi um fator 
decisivo para o bom desenvolvimento do projeto e que o uso de softwares alinhados com a metodologia es teve presente em todos os trabalhos entregues, 0 que evidencia tendência de trabalho com esta metodologia pelos estudantes.

Palavras-chave: Ensino-aprendizagem. Projetos. Inovação. Engireenig. Building Information Modelling. 


\section{A PROPENSÃO AO BIM NO CURSO DE ENG. CIVIL DURANTE ATIVIDADES REMOTAS NA PANDEMIA}

\section{INTRODUÇÃO}

Cada vez mais é observado na indústria da arquitetura, engenharia e construção (AEC) um aumento de complexidade nos projetos, que demandam conhecimentos interdisciplinares e maior integração entre os diversos atores de uma cadeia produtiva. Tal cenário se beneficia da metodologia de trabalho em BIM, que é capaz de possibilitar a integração entre os diversos agentes da construção (LEITNER et al., 2018).

A Modelagem da Informação da Construção é um dos mais promissores desenvolvimentos na AEC (EASTMAN et al., 2014). Porém, a incorporação do conceito de BIM na formação de engenheiros civis e arquitetos ainda encontra barreiras (LEITNER et al., 2018). A maioria das experiências nacionais abordam o ensino do BIM apenas em disciplinas isoladas, o que tornam ausentes os conceitos de coordenação, integração e colaboração, sendo estes essenciais para uma prática de projeto baseada no BIM (RUSHEL; ANDRADE; MORAIS, 2013).

Assim, fica evidente a necessidade de formação de profissionais cada vez mais alinhados as expectativas do mercado (KEHL et al., 2018), que hoje tem no BIM uma importante alavanca para a melhoria da qualidade e produtividade no setor (DANTAS FILHO et al., 2017). Nesse contexto, as instituições de ensino têm papel fundamental por ser responsável principal pela formação de graduação, mas que ainda detém foco restrito à habilidades técnicas ( hard skills), deixando as chamadas habilidades sociais (soft skills) como secundárias ou até mesmo negligenciadas (MARTINS; MONTEIRO; FERREIRA, 2020).

Tais desenvolvimentos tornaram-se críticos em virtude dos riscos da pandemia do novo Coronavírus (SARS-CoV-2) que impôs uma nova realidade para as diferentes organizações (ALI; ALHARBI, 2020) com fechamento temporário de empresas, quarentena e restrições à mobilidade (OECD, 2020). Tais efeitos também produziram rearranjos e desafios no ensino que passou a usar mais intensivamente as Tecnologias de Informação e Comunicação (TIC) (SEPASGOZAR, 2020).

É nesse contexto de desafios que o trabalho se encaixa, uma vez que o BIM permite o trabalho remoto e promove a colaboração entre os agentes envolvidos em um projeto, cuja questão de pesquisa foi: qual a propensão de alunos de engenharia civil de curso presencial à adoção do BIM no desenvolvimento de trabalhos remotos? Assim, este trabalho teve por objetivo analisar a propensão ao trabalho com BIM por alunos de graduação em engenharia civil. Tomou-se como objeto de estudos a elaboração de um projeto residencial desenvolvido remotamente no âmbito de uma disciplina em um curso de uma universidade federal no interior do estado do Ceará.

A seguir, apresenta-se o referencial teórico com enfoque no BIM no ensino e as competências inerentes à sua utilização. Em seguida, apresenta-se o método de pesquisa que consistiu de um estudo de caso em uma universidade federal no estado do Ceará. Os resultados e discussões são apresentados na sequência, seguido das conclusões e referências ao final.

\section{REFERENCIAL TEÓRICO}

A Modelagem da Informação da Construção (Building information Modelling - BIM) 
pode ser conceituado como um conjunto de processos, políticas e tecnologias que permitem o gerenciamento de um modelo virtual de informações de uma construção (SUCCAR, 2009). Tal modelo virtual pode contemplar todo o seu ciclo de vida, e servir a diversas finalidades. O uso da metodologia BIM representa uma mudança de paradigmas, saindo da lógica de projeto individual e fragmentado para o trabalho colaborativo e continuado (EASTMAN et al., 2014).

Com a crescente adoção do BIM no mercado de trabalho, as instituições de ensino têm tentado inserir em seus cursos de AEC. Porém sua adesão ainda é lenta e pontual, o que pode comprometer alguns aspectos de colaboração inerentes, caracterizando o atual cenário como introdutório (RUSCHEL; ANDRADE; MORAIS, 2013).

Neste contexto, o BIM promove o desenvolvimento de 3 tipos de competências gerais individuais, são elas essenciais, de Domínio e de Execução (SUCCAR; SHER; WILLIAMS, 2013). De acordo com os autores, as chamadas competências essenciais dizem respeito às habilidades pessoais que os permitem conduzir atividades. Já as competências de Domínio correspondem às habilidades profissionais, que consistem na metodologia utilizada para entregar um resultado. Por último, as competências de Execução estão relacionadas às habilidades ferramentais ou técnicas.

Além dessas competências individuais, também é possível inserir as chamadas Competências Transversais ou Sociais, que estão ligadas ao trabalho colaborativo. Para essa categoria, são listados diversos tipos de habilidades, como gerenciamento, colaboração, negociação, liderança e gestão de conflitos. Tais competências são primordiais para promover um ambiente colaborativo e integrado, realidade cada vez mais presente (AGUILAR E PEREIRA, 2017).

Tais competências podem catalisar a formação em engenharia civil, especialmente frente às nodas Diretrizes Curriculares Nacionais que preconiza a formação por competência (BRASIL, 2018). Sendo assim, interessa investigar a propensão - ou seja, a inclinação ou facilidade que os estudantes possuem para adquirir essas competências, e a prontidão - ou seja, a habilidade inata dos estudantes.

\section{MÉTODO DE PESQUISA}

Após revisão da literatura sobre o BIM e sua implementação no ensino de Arquitetura e Engenharia, realizou-se um estudo com estudantes, na Universidade Federal do Ceará, Campus Crateús. Tomou-se como objeto de estudos a elaboração de um projeto residencial desenvolvido remotamente no âmbito da disciplina de Projeto e Construção de Edifícios 1, 5을 semestre na estrutura curricular.

O trabalho proposto consistiu no desenvolvimento de um projeto arquitetônico residencial que seria avaliado com os mesmos parâmetros de avaliação para aprovação na prefeitura da cidade em questão, tomando como base o Plano Diretor e o Código de Obras e Posturas. Os alunos foram divididos em equipes e cada equipe recebeu um terreno e programa de necessidades diferente. As equipes desenvolveram seus trabalhos remotamente, em virtude da pandemia de corona vírus que provocou a suspensão das atividades presenciais na universidade.

Ao término do trabalho, realizou-se uma pesquisa com os alunos por meio de questionário on-line, contando com 17 perguntas objetivas. Dos 22 estudantes matriculados, 19 responderam a pesquisa (86,4\% da população). Os dados foram 
analisados quantitativamente, por meio de estatísticas descritivas como média e desvio padrão e são apresentados na próxima seção.

\section{$4 \quad$ RESULTADOS E DISCUSSÕES}

A Figura 1 apresenta os softwares utilizados para o projeto, bem como se os alunos já possuiam formação em cada um deles.

Figura 1 - Softwares utilizados no desenvolvimento gráfico do trabalho

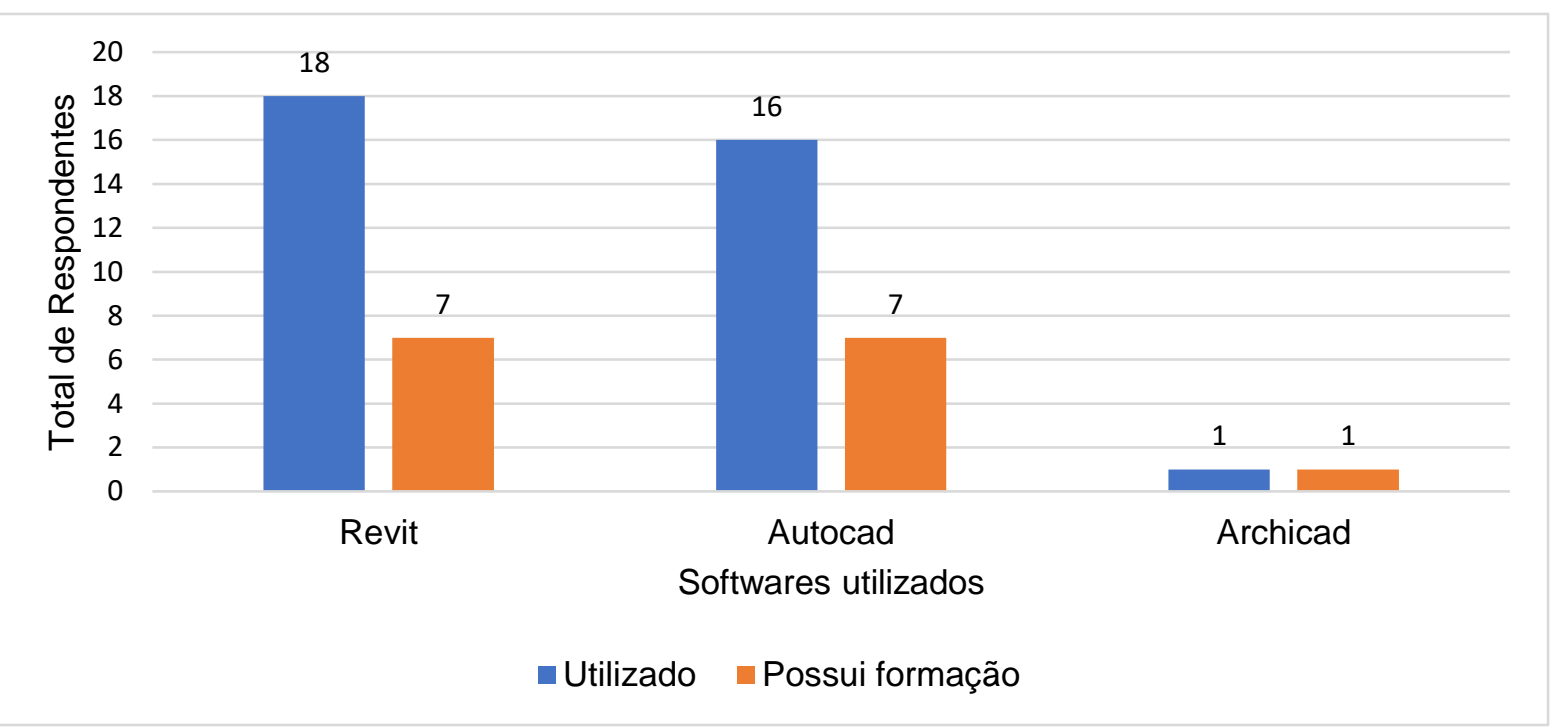

Fonte: Dos autores (2021)

Pode-se observar que majoritariamente o software Revit foi o software BIM mais aplicado. Porém, poucos dos alunos tinham formação específica no mesmo. Além disso, é possível notar que, embora esse software tenha sido aplicado, o Autocad também foi amplamente utilizado o que indica insegurança para realizar o trabalho completo no Revit ou ainda é reflexo da baixa formação demonstrada no referido software. É importante salientar que com a entrega dos trabalhos foi possível observar que todas as equipes utilizaram o Revit ou o Archicad em pelo menos alguma parte do trabalho.

A Tabela 1 apresenta se o trabalho da disciplina incentivou o uso de tais softwares, haja vista que é feita uma exposição sobre o processo de projeto tradicional e em BIM, sem nenhum desenvolvimento executivo.

Tabela 1 - Incentivo do projeto quanto ao uso dos softwares.

\begin{tabular}{c|c|c}
\hline $\begin{array}{c}\text { O trabalho incentivou o primeiro contato com o software } \\
\text { escolhido }\end{array}$ & \multicolumn{2}{c}{$\begin{array}{c}\text { Respondentes } \\
\text { Porcentagem } \\
\text { relativa }\end{array}$} \\
\hline Sim, foi o primeiro contato & 5 & $26,3 \%$ \\
Não, mas motivou o aperfeioamento nos softwares & 13 & $68,4 \%$ \\
Não, mas não motivou o aperfeiçoamento nos softwares & 1 & $5,3 \%$ \\
Total & $\mathbf{1 9}$ & $\mathbf{1 0 0 \%}$ \\
\hline
\end{tabular}

Fonte: Dos autores (2021) 
Pode-se constatar a prontidão que os estudantes já possuiam para realizar o trabalho, dado que cerca de $70 \%$ afirmou que não foi o primeiro contato, mas que o trabalho motivou o aperfeioamento nos softwares

A Tabela 2 relaciona os fatores que motivaram os alunos a escolherem os softwares para o desenvolvimento do projeto.

Tabela 2 - Motivação para a escolha dos softwares

\begin{tabular}{|c|c|c|}
\hline Motivação para a escolha & Respondentes & $\begin{array}{l}\text { Porcentagem } \\
\text { relativa }\end{array}$ \\
\hline Familiaridade com o(s) software(s) & 5 & $89,5 \%$ \\
\hline Desconhecimeno de outras metodologias & 13 & $10,5 \%$ \\
\hline Adaptação ao mercado & 1 & $57,9 \%$ \\
\hline Facilidade de visualização do que é projetado & 19 & $73,7 \%$ \\
\hline
\end{tabular}

Fonte: Dos autores (2021)

Foi possível perceber que grande parte dos respondentes possui algum grau de familiaridade com os softwares. Isso demonstra o interesse dos alunos nas novas tecnologias e metodologias de trabalho. A facilidade de visualização ao longo do projeto, motivada por um maior dinamismo, além da possibilidade de extrair informações construtivas, como materiais, índices, quantitativos, ou ainda informações como custos ou tempo também foram motivadores. Como já mencionado, já existe uma procura por profissionais voltados à metodologia BIM, o que é perceptível aos alunos ainda na graduação, o que também os motiva a adotá-lo mesmo que não seja algo imperativo.

A Figura 2 apresenta um comparativo entre o que era esperado e o que foi obtido pelos respondentes com a utilização dos softwares escolhidos.

Figura 2 - Comparativo entre o esperado e o obtido com a utilização dos softwares

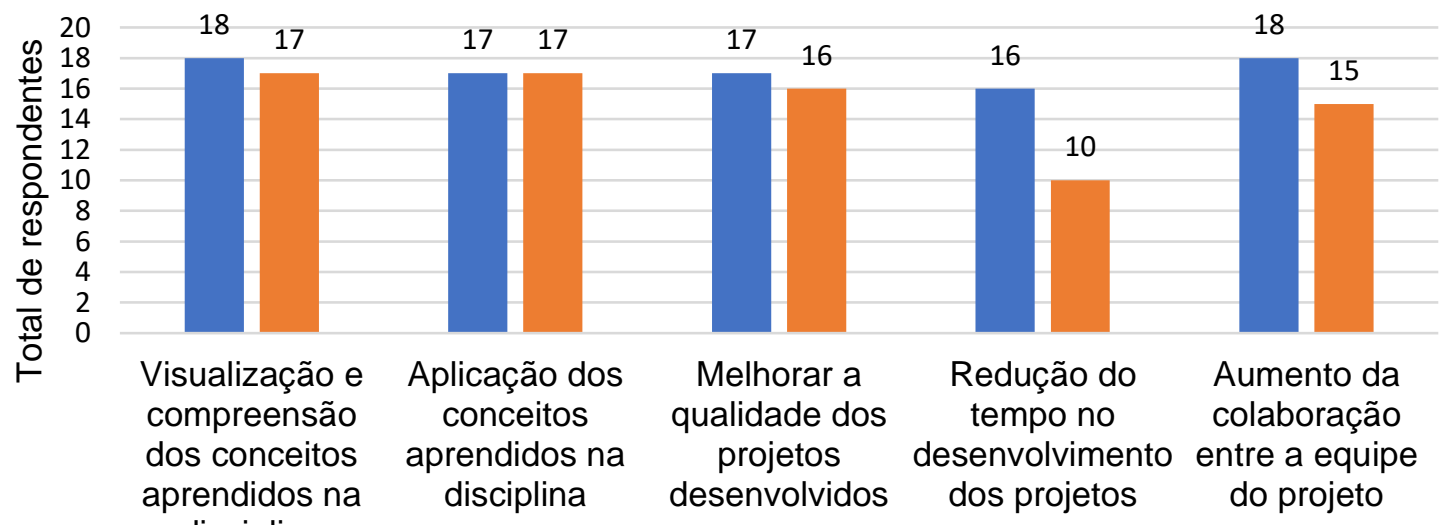
disciplina

$$
\text { - Esperado } \quad \text { Obtido }
$$

Fonte: Dos autores (2021) 
Apesar de alguns pontos divergentes, de modo geral as contribuições foram atingidas pelos respondentes de maneira satisfatória. Pode-se observar que as expectativas dos respondentes quanto à utilização dos softwares estão atreladas às expectativas quando ao uso de uma metodologia BIM por si mesma. Algumas reflexões podem ser realizadas ao se observar os objetivos menos atingidos. Quanto à colaboração da equipe, pode-se depreender que não só atributos ferramentais (relacionas ao software ou a internet por exemplo) podem ser importantes, mas também o engajamento e interação da própria equipe, um desafio presente mesmo antes da necessidade de trabalho remoto. Quanto a redução do tempo de projeto, é uma expectativa comum com a metodologia BIM. Mas é importante perceber que em geral, o tempo de projeto é maior, pois busca-se identificar e corrigir os problemas construtivos ainda na fase de projetos, para evitar problemas e custos adicionais nas fases seguintes. No caso do trabalho proposto, a única fase abordada é justamente a de desenvolvimento de projeto.

A Tabela 3 apresenta os motivos para não atingir os objetivos desejados.

Tabela 3 - Motivos para a não obtenção dos resultados desejados

\begin{tabular}{|c|c|c|}
\hline Motivos & Respondentes & $\begin{array}{l}\text { Porcentagem } \\
\text { relativa }\end{array}$ \\
\hline $\mathrm{O}$ arquivo muito pesado para o computador & 8 & $42,1 \%$ \\
\hline Os objetivos foram alcançados & 7 & $36,8 \%$ \\
\hline Habilidade com o software foi insuficiente & 6 & $31,6 \%$ \\
\hline $\begin{array}{l}\text { A conexão com a internet dificultou o compartilhamento de } \\
\text { arquivos }\end{array}$ & 3 & $15,8 \%$ \\
\hline $\begin{array}{c}\text { A maioria dos membros da equipe não dominavam o } \\
\text { software principal }\end{array}$ & 1 & $5,3 \%$ \\
\hline
\end{tabular}

Fonte: Dos autores (2021)

É compreensível que o tamanho do arquivo seja um obstáculo presente no trabalho, principalmente quando as diversas informações sendo inseridas em algum modelo, ou o uso de mais de um software durante o projeto. Esse fator também é agravado pela necessidade de compartilhamento de tais modelos e informações, o que leva a outro item, as dificuldades provenientes da conexão com a internet. Com a dispensa das aulas presenciais, muitos alunos voltaram para suas cidades e muitos enfrentaram problemas quando à cobertura de internet disponível.

Apesar da familiaridade assumida pelos alunos, outro fator significativo foi a habilidade com software. Isto pode estar relacionado ao fato de o projeto precisar ser desenvolvido desde seu início, situação mais próxima da realidade do trabalho. Já emcursos voltados para aprendizagem de softwares de desenho e modelagem, o projeto normalmente já está feito, cabendo apenas o aprendizado da ferramenta na modelagem, ou seja, um conhecimento unicamente ferramental.

Os meios de comunicação utilizados foram aplicativos de mensagens instantâneas (Whatsapp, Telegram, etc.) (100\% dos respondentes), webconferências (100\%) e e-mail (53\%), cujos objetivos de interação foram apresentados na Tabela 4. 
Tabela 4 - Objetivos das interações entre os membros da equipe

\begin{tabular}{c|c|c}
\hline \multicolumn{1}{c}{ Objetivos } & \multicolumn{1}{c}{ Respondentes } & $\begin{array}{c}\text { Porcentagem } \\
\text { relativa }\end{array}$ \\
\hline $\begin{array}{c}\text { Resolução de dúvidas em partes interdependentes do } \\
\text { trabalho }\end{array}$ & 17 & $89,5 \%$ \\
$\begin{array}{c}\text { Unir as partes individuais desenvolvidas ao longo de um } \\
\text { determinado período }\end{array}$ & 15 & $78,9 \%$ \\
Correção de pontos específicos no trabalho \\
Realização das atividades em conjunto & 14 & $73,7 \%$ \\
\hline
\end{tabular}

Fonte: Dos autores (2021)

Pode-se observar que as interações promoveram o trabalho colaborativo e a união de desenvolvimentos individuais, aspectos inerentes ao desenvolvimento de projetos em BIM. Em relação aos objetivos das interações síncronas, especialmente das webs conferências entre os membros da equipe, existe uma mescla de objetivos. Estes estão muito ligados a como os alunos decidiram dividir suas responsabilidades entre si. Pode-se observar que houve divisão de partes de trabalho, mas também houve por mais da metade dos alunos atividades em conjunto, em que a colaboração em tempo real entre os membros servia para a realização de uma atividade comum.

Dez respondentes afirmaram ter realizado o envio de múltiplas versões de um mesmo arquivo a cada atualização, enquanto o compartilhamento em nuvem com atualização automática de um único arquivo foi superior (12 respondentes). Como os alunos não tinham apenas um produto a entregar no trabalho, então as duas modalidades de compartilhamento foram utilizadas, algumas vezes de maneira não exclusiva. É interessante perceber que maior parte dos respondentes utilizou a atualização em nuvem, uma vantagem em nível de comunicação, de espaço, organização e de colaboração entre os membros.

A Tabela 5 apresenta a avaliação da comunicação no desenvolvimento do trabalho.

Tabela 5 - Classificação da comunicação adotada

\begin{tabular}{c|c|c}
\hline \multicolumn{1}{c}{ Classificação } & \multicolumn{1}{c}{ Respondentes } & \multicolumn{1}{c}{$\begin{array}{c}\text { Porcentagem } \\
\text { relativa }\end{array}$} \\
\hline $\begin{array}{c}\text { Falha, a pouca comunicação impactou negativamente no } \\
\text { resultado final }\end{array}$ & 0 & $0,0 \%$ \\
$\begin{array}{c}\text { Intermediária, o resultado poderia ser otimizado com um } \\
\text { maior nível de comunicação }\end{array}$ & 5 & $26,3 \%$ \\
$\begin{array}{c}\text { Boa, o trabalho foi desenvolvido com um bom nível de } \\
\text { colaboração }\end{array}$ & 8 & $42,1 \%$ \\
$\begin{array}{c}\text { Excelente, o fluxo de informações eracontínuo entre as } \\
\text { partes envolvidas } \\
\text { Total }\end{array}$ & 6 & $31,6 \%$ \\
\hline
\end{tabular}


Fonte: Dos autores (2021)

Grande parte dos respondentes classificou a comunicação como boa ou excelente, indicando um bom nível de colaboração, ou até mesmo um fluxo contínuo de informações entre as partes. Nenhum respondente indicou que a comunicação impactou negativamente o resultado final, apesar de alguns respondentes indicaram que o resultado do trabalho poderia ser otimizado com uma melhor comunicação.

Pode-se verificar, na Tabela 6, as principais causas atribuídas para as dificuldades de comunicação.

Tabela 6 - Motivos atribuídos para as dificuldades de comunicação

\begin{tabular}{c|c|c}
\hline Dificuldades & Respondentes & Porcentagem relativa \\
\hline Conexão com internet & 10 & $52,6 \%$ \\
Plano de telefonia & 2 & $10,5 \%$ \\
Cobertura de telefonia & 3 & $15,8 \%$ \\
Engajamento da equipe & 4 & $21,1 \%$ \\
Não houveram dificuldades & 6 & $31,6 \%$ \\
\hline
\end{tabular}

Fonte: Dos autores (2021)

Pode-se observar que $31,6 \%$ dos alunos não relataram nenhum tipo de dificuldade, enquanto a maioria respondeu enfrentar dificuldades com a internet, que pode ter impactado tanto a comunicação quanto outros aspectos do trabalho, como foi visto anteriormente. Também é perceptível que muitos alunos relataram entre as dificuldades o engajamento da equipe, o que mostra que a comunicação não pode ser observada apenas pelos meios de comunicação usados. É importante perceber que mesmo com implementações tecnológicas ou de novos processos e metodologias ainda é necessário que a participação ativa das partes envolvidas seja efetiva para a boa realização de um trabalho. Um fator positivo que essa dificuldade foi pequena comparada a outros fatores, contudo significativa.

A Tabela 7 apresenta os tipos de ajustes necessários durante a realização do projeto. Estes ocorreram através de alterações nas dimensões de ambientes, edificação e loteamento, em função de índices geométricos e da legislação municipal.

Tabela 7 - Ajustes durante o projeto

\begin{tabular}{|c|c|c|}
\hline Ajustes & Respondentes & $\begin{array}{l}\text { Porcentagem } \\
\text { relativa }\end{array}$ \\
\hline $\begin{array}{c}\text { Dimensões dos ambientes, em função do Código de Obras e } \\
\text { posturas }\end{array}$ & 13 & $68,4 \%$ \\
\hline $\begin{array}{l}\text { Dimensões dos ambientes, em função dos índices } \\
\text { geométricos para otimizar o projeto }\end{array}$ & 13 & $68,4 \%$ \\
\hline
\end{tabular}


Dimensões da edificação, em função dos índices geométricos para otimizar o projeto

Dimensões da edificação, em função da Lei de Uso e Ocupação dos Solos

Dimensões no loteamento, em função da Lei de Uso e Ocupação dos Solos
10

$52,6 \%$

11

$57,9 \%$

11

$57,9 \%$

Nenhum. Não foi feito nenhum reajuste

Fonte: Dos autores (2021)

Pode-se notar uma aproximação do trabalho com a realidade de projeto, considerando que nenhuma equipe relatou não necessitar fazer reajustes ao longo do desenvolvimento. Tais ajustes são importantes para se adequar a demandas específicas de clientes, legislação construtiva, como as mencionadas anteriormente, ou à índices de otimização de projeto, por exemplo. O tempo gasto para esses ajustes é apresentado na Tabela 8.

Tabela 8 - Tempo de ajustes

\begin{tabular}{c|c|c}
\hline Tempo de ajustes & \multicolumn{1}{c}{ Respondentes } & $\begin{array}{c}\text { Porcentagem } \\
\text { relativa }\end{array}$ \\
\hline Nenhum. Não foi feito nenhum tipo de reajuste & 0 & $0,0 \%$ \\
$\begin{array}{c}\text { Pouco. Os ajustes necessários eram pequenos e simples de } \\
\text { resolver }\end{array}$ & 8 & $42,1 \%$ \\
$\begin{array}{c}\text { Médio. Quantidade considerável de ajustes que consumiram } \\
\text { bastante tempo de desenvolvimento de projeto. }\end{array}$ & 11 & $57,9 \%$ \\
$\begin{array}{c}\text { Muito tempo. O projeto teve de ser quase todo refeito } \\
\text { Total }\end{array}$ & 0 & $0,0 \%$ \\
\hline
\end{tabular}

Fonte: Dos autores (2021)

Mesmo que mais da metade dos alunos tenham relatado haver uma grande quantidade de ajustes, consumindo bastante tempo, não houve casos de que o projeto teve de ser refeito. Além disso, é importante perceber que cada equipe tinha terrenos e propostas de projeto diferentes, distribuídas aleatoriamente, o que pode ter influenciado para a necessidade de maior ou menor quantidade de ajustes. A habilidade dos alunos, bem como a quantidade de alunos responsáveis por esses ajustes em cada equipe, pode ter sido também relevante para a quantidade de tempo utilizado.

O tempo de ajustes também é um fator que se beneficia da metodologia BIM, uma vez que esta proporciona uma maior facilidade em identificar e corrigir erros de projeto. A utilização da atualização em nuvem de um único modelo, utilizada por parte dos alunos, também otimiza esse processo, evitando que uma mesma modificação tenha que ser reproduzida em diversos arquivos diferentes.

A Tabela 9 apresenta a percepção do nível de clareza do projeto desenvolvido. 
Tabela 9 - Percepção do nível de clareza do projeto desenvolvido

\begin{tabular}{c|c|c}
\hline Classificação & \multicolumn{1}{c}{$\begin{array}{c}\text { Respondentes } \\
\text { Porcentagem } \\
\text { relativa }\end{array}$} \\
\hline $\begin{array}{c}\text { Excelente, apresentou-se detalhes que ampliaram a clareza } \\
\text { do projeto }\end{array}$ & 6 & $31,6 \%$ \\
Satisfatório, os elementos exigidos estavam claros & 12 & $63,2 \%$ \\
Insuficiente, alguns elementos exigidos não estavam claros \\
Péssimo, alguns elementos exigidos não foram \\
apresentados \\
Total & 1 & $5,3 \%$ \\
\end{tabular}

Fonte: Dos autores (2021)

Verifica-se que os estudantes ficaram satisfeitos com o nível de clareza. Isto foi corroborado pela avaliação da disciplina, uma vez os modelos 3D possibililtaram ampla compreensão da proposta realizada.

A Figura 3 apresenta a divisão das tarefas nas equipes, com a quantidade de integrantes envolvidos em cada tarefa.

Figura 3 - Divisão de tarefas por equipe

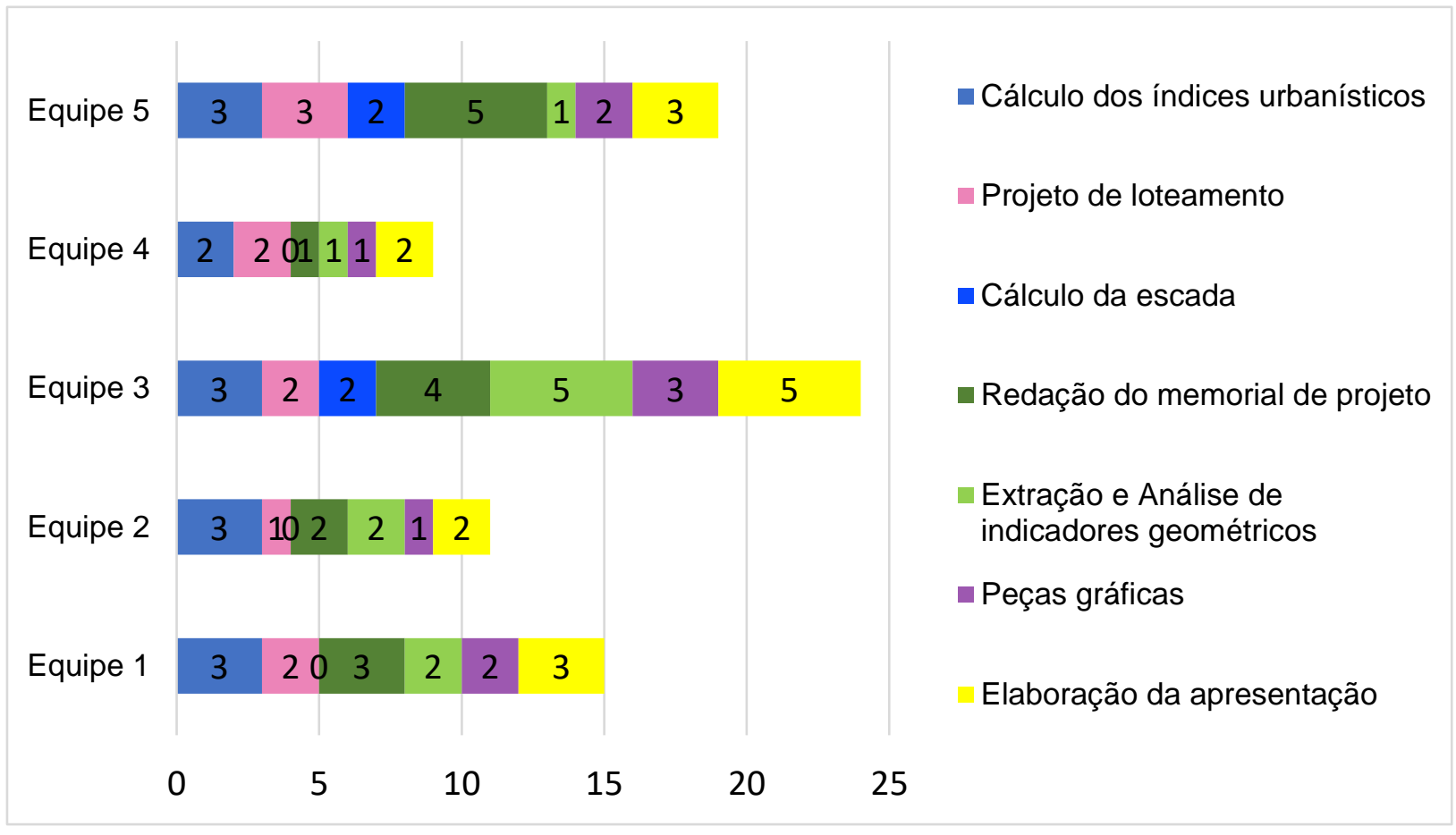

Fonte: Dos autores (2021)

A divisão de responsabilidades nas equipes fica evidente. Dentre as muitas funções, pode-se observar que há tarefas em que todos os integrantes participam, enquanto outras 
que são exclusivas de determinados membros. Isso é importante para observar a cooperação entre os membros no desenvolvimento do projeto. As divisões podem ter sido feitas por nível de dificuldade, por aptidão ou interesse individual, em caso de atividades exclusivas, ou tarefas compartilhadas, que seriam desenvolvidas em conjunto (com 2 ou mais integrantes). Isso demonstra o dinamismo e a colaboração das equipes para que as habilidades dos membros fossem aproveitadas ao máximo, bem como respeitar o limite de responsabilidades que cada um poderia se comprometer.

\section{CONSIDERAÇÕES FINAIS}

Este trabalho teve por objetivo analisar a propensão ao trabalho com BIM por alunos de graduação em engenharia civil. Para tanto, tomou-se como objeto de estudos a elaboração de um projeto residencial desenvolvido remotamente no âmbito de uma disciplina em um curso de uma universidade federal no interior do estado do Ceará.

Observou-se que a comunicação, elemento essencial para a integração em um processo de aplicação da metodologia BIM, desempenhou um papel decisivo nos resultados do trabalho. Verificou-se, também, forte alinhamento dos alunos à metodologia BIM, demonstrado pela escolha de ferramentas, processos de desenvolvimento e pelo trabalho colaborativo. Com relação à visão dos estudantes, torna-se perceptível grande interesse na adequação a novas tecnologias e metodologias, especialmente o BIM. Estes resultados evidenciam a tendência de trabalho com esta metodologia pelos estudantes.

O estudo possui algumas limitações como ter se pautado na percepção dos estudantes sobre 0 trabalho desenvolvimento, o que tentou ser dirimido com as observações realizadas durante as apresentações e nas avaliações dos projetos na disciplina e que não diminuem as reflexões aqui realizadas. Estudos futuros poderiam averiguar formas de avaliação mais contundentes e objetivas para tal. Ainda, pode-se realizar experimentos separando as equipes em dois grupos, com e sem BIM, e verificar a facilidade e a qualidade do desenvolvimento do projeto em causa.

\section{Agradecimentos}

Os autores agradecem a Pró-reitoria de Graduação pela concessão de bolsa de iniciação à docência durante o ano de 2020, especialmente a sua prorrogação devido à pandemia de coronavírus.

\section{REFERÊNCIAS}

ALI, I.; ALHARBI, O. M. L. COVID-19: Disease, management, treatment, and social impact. Science of the Total Environment, v. 728, p. 138861, 2020.

BRASIL. Resolução no 7 MEC/CNE/CES, de 18 de dezembro de 2018. Estabelece as Diretrizes para a Extensão na Educação Superior Brasileira e regimenta o disposto na Meta

12.7 da Lei no 13.005/2014, que aprova o Plano Nacional de Educação - PNE 2014-2024 e dá

outras providências. Disponível em: < http://www.in.gov.br/materia/-

/asset_publisher/Kujrw0TZC2Mb/content/id/55877808 > Acessado em: 30 abr. 2020 
DANTAS FILHO, J. B. P.; BARROS NETO, J. DE P.; ANGELIM, B. M. Mapeamento do fluxo de valor de processo de construção virtual baseado em BIM. Ambiente Construído, v. 17, n. 4, p. 343-358, dez. 2017.

EASTMAN, C. et al. BIM Handbook: a guide to Building Information Modeling for owners, managers, designers, engineers, and contractors. Hoboken: John Wiley \& Sons, 2008. $490 \mathrm{p}$.

KEHL, C., STUMPF, C. D., ROSA, D. C., SILVA, A. F., ISATTO, L. E. Ensino de BIM em um curso de Arquitetura e Urbanismo: avaliação da percepção de demanda. In: ENCONTRO NACIONAL DE TECNOLOGIA DO AMBIENTE CONSTRUÍDO, 17., 2018, Foz do Iguaçu. Anais... Porto Alegre: ANTAC, 2018.

LEITNER, Drielle S. et al. PROPOSTA DE PLANO DE EXECUÇÃO BIM EM UMA EMPRESA JÚNIOR DE ENGENHARIA CIVIL: UMA ALTERNATIVA PARA A INTRODUÇÃO DE BIM NA FORMAÇÃO EM ENGENHARIA. In: CONGRESSO BRASILEIRO DE EDUCAÇÃO EM ENGENHARIA, 46., Salvador. Anais ... Brasília: ABENGE, 2018. Disponível em:

$<$ http://www.abenge.org.br/sis artigo doi.php?e=COBENGE\&a=18\&c=1041>

MARTINS, T. G. M; MONTEIRO, N. P; FERREIRA, G. S. A formação do engenheiro do futuro: as contribuições do movimento empresa júnior. In: CONGRESSO BRASILEIRO DE EDUCAÇÃO EM ENGENHARIA, 48., Caxias do Sul. Anais... Brasília: ABENGE, 2020. Disponível em: <https://doi.org/10.37702/COBENGE.2020.3117>

OECD. Evaluating the initial impact of COVID-19 containment measures on economic activity Introduction and key messages. Tackling coronavirus contributing to a global effort, June, p. 1-5, 2020. Disponível em:

<https://www.oecd.org/coronavirus/policy-responses/evaluating-the-initial-impactof-covid-19-containment-measures-on-economic-activity-b1f6b68b/>.

RUSCHEL, R. C.; ANDRADE, M. L. V. X. de; MORAIS, M. de. O ensino de BIM no Brasil: onde estamos? Ambiente Construído, v. 13, n. 2, p. 151-165, 2013.

SEPASGOZAR, S. M. E. Digital twin and web-based virtual gaming technologies for online education: A case of construction management and engineering. Applied Sciences (Switzerland), v. 10, n. 13, 2020.

SUCCAR, B.; SHER, W.; WILLIAMS, A. An integrated approach to BIM competency assessment, acquisition and application. Automation in Construction, v. 35, p. 174189, nov. 2013. Disponível em:

<http://linkinghub.elsevier.com/retrieve/pii/S0926580513000836>.

\section{THE PROPENSITY TO BIM USES IN CIVIL ENGINEERING COURSE DURING REMOTE ACTIVITIES IN THE PANDEMIC}

Abstract: The Building Information Modeling (BIM) promotes the digitization of construction information for different uses throughout the project life cycle. Its application demands new skills of professionals in Architecture, Engineering and Construction (AEC). This requirement stimulates the necessity for adjustments in the curriculum of graduation courses in AEC, which have been taking place gradually and inefficiently. Especially in the year 2020, 
marked by a global pandemic, the academic community needed to adapt to teaching remote, increasing the requirement of collaboration among students in group activities of assessment. Face of this, its highlight that BIM has collaborative work tools that can support remote and collaborative work. Thus, this work aimed to investigate the inclination to work with BIM with undergraduate students in civil engineering. For that, it took as the object of study the elaboration of a residential design, developed remotely in the scope of a discipline ongoing of a Federal University in the state of Ceará, Brazil. An online survey was applied after conclusion of the activity in question, and it was possible to understand the students' challenges in the process, especially about BIM implementation and the dynamics of collaboration. It was found that communication was a crucial factor to the good development of the design, and the use of software aligned with the methodology was present in all designs delivered, which shows a tendency for students to work with this methodology.

Keywords: Engireenig. Building Information Modelling. 